\title{
Improving vascular health: are pills the answer?
}

\author{
Lowering the statin treatment threshold raises important questions
}

\author{
Liam Smeeth professor of clinical epidemiology ${ }^{1}$, Harry Hemingway professor of clinical epidemiology ${ }^{2}$
}

'London School of Hygiene and Tropical Medicine, London WC1E 7HT, UK; ${ }^{2}$ University College London, London, UK

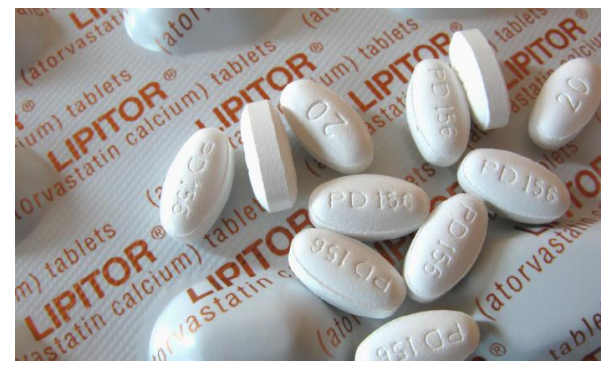

[Image: C \& M WERNER, VISUALS UNLIMITED/SPL]

Guidelines from the National Institute for Health and Clinical Excellence, ${ }^{1}$ the United States, ${ }^{2}$ and Europe ${ }^{3}$ recommend statins for people with cardiovascular disease and for those at high risk of developing disease on the basis of an individual risk assessment. Although there has been much debate about the optimal risk assessment tool, surprisingly, the risk threshold above which statins are recommended has been less hotly debated. A risk of developing cardiovascular disease of greater than $20 \%$ over 10 years-or greater than $10 \%$ over five years-is widely used as the cut-off point for prescribing statins. The results of a recent meta-analysis of data from the Cholesterol Treatment Trialists Collaborators directly challenges the consensus over this threshold. ${ }^{4}$

Statins reduce the relative risk of major vascular events by around $20 \%$ per $1 \mathrm{mmol} / \mathrm{L}$ reduction in low density lipoprotein-cholesterol-the typical effect on lipids produced by a low to moderate dose of statin. The striking finding from the new meta-analysis is that the established beneficial effects were seen across a broad spectrum of vascular risk, including people with a five year risk of vascular disease of less than $5 \%$, well below the current threshold for statin treatment. Therefore, people at low to medium risk of disease, who are seldom currently prescribed a statin, may be missing out on a treatment that has been proved to reduce the incidence of future vascular events. Should clinical guidelines now be changed and statins recommended for a larger proportion of the population?

We suggest four factors that warrant detailed consideration before such a revolutionary change in guidance is made. Firstly, cost is a consideration. When initially shown to be effective, statins had 10 year patents and were expensive. Even though relative measures of benefit (such as the rate ratio) are similar across different groups, absolute measures of benefit (such as numbers of events prevented) vary by baseline risk. It is cheaper to prevent one heart attack by treating 100 people at very high risk of vascular disease than by treating 1000 people at low risk. However, as patents expire, cheap generic versions of statins have become available, including most recently atorvastatin. ${ }^{5}$ Although it may be relatively cheap to widen the use of statins use now, a rigorous cost-effectiveness analysis of this approach is yet to be completed.

Secondly, statins cause harms. Myopathy, and the more serious rhabdomyolysis, are important, if uncommon, adverse effects $\left(0.1 / 1000\right.$ people treated for a year or less). ${ }^{67}$ Statins increase the risk of haemorrhagic stroke, ${ }^{4}$ although the numbers of ischaemic strokes prevented outweighs the increased risk of haemorrhagic stroke. There has been great concern that statins may increase the risk of diabetes. ${ }^{8}$ A small increased risk seems likely, but again, the known benefits of statins on vascular outcomes probably outweigh this risk in all groups studied so far. Previous concerns that statins may increase the risk of cancer have been contradicted by evidence from large scale observational studies and trials. ${ }^{7}$ However, the potential for increased rates of harm with increased statin use cannot be ignored, and ongoing vigilance for adverse effects is needed.

A third important factor relevant to any discussion of widening the use of statins is the viewpoint of the patient, and unanswered questions abound. For patients to decide whether or not to take statins more information is needed on outcomes that are relevant to patients. A patient may ask, "Will statins extend my life?" This intuitive measure of impact is rarely reported and may give surprising results. For example, even among high risk patients undergoing coronary artery bypass grafting compared with medical therapy, surgery extended survival at 10 years by only about 35 weeks. ${ }^{10}$ Extending life by a moderate amount may be important for some patients but not for others. Do patients want to delay vascular events? The answer is probably "yes."

However, death is inevitable and when it comes some people may prefer a sudden fatal vascular event to a more drawn out 
alternative. Do patients on lifelong statin treatment have better quality of life? We don't know for sure.

Lastly, why take pills to treat the ills of society? Vascular events occur largely because of the consequences of industrialised living associated with poor diet, low levels of exercise, and smoking. These are (potentially) modifiable factors. Sustainable improvements in human health-not restricted to vascular disease and accessible to rich and poor alike — could be achieved by improving diets, encouraging physical activity, and reducing smoking. The alternative scenario- of whole populations taking a daily tablet to mitigate against unhealthy lifestyles - is far from attractive. Even the consideration of this option may suggest that the medical profession is losing its way in efforts to allow people to live healthier longer lives. If we developed a tablet that prevented the adverse effects of smoking, would we advocate widespread consumption, forgetting that a better aim would be to stop people smoking in the first place?

Evidence that a drug treatment reduces the relative risk for vascular events is noteworthy but not sufficient to warrant revising current treatment thresholds. There is a danger that the quest for a healthier world is replaced by a quest to get more and more people taking tablets.

Competing interests: Both authors have completed the ICMJE uniform disclosure form at www.icmje.org/coi_disclosure.pdf (available on request from the corresponding author) and declare: LS is supported by a senior clinical fellowship from the Wellcome Trust; no financial relationships with any organisations that might have an interest in the submitted work in the previous three years; no other relationships or activities that could appear to have influenced the submitted work.

Provenance and peer review: Commissioned; not peer reviewed.

1 National Institute for Health and Clinical Excellence. Lipid modification. CG67. 2010. www. nice.org.uk/CG67.

2 National Heart Lung and Blood Institute. ATP III update 2004. www.nhlbi.nih.gov/guidelines/ cholesterol/atp3upd04.htm.
3 Perk J, De Backer G, Gohlke H, Graham I, Reiner Ž, Verschuren M, et al. European Guidelines on cardiovascular disease prevention in clinical practice (version 2012). Eur Heart J2012; published online 3 May. http://eurheartj.oxfordjournals.org/cgi/doi/10.1093/ eurheartj/ehs092.

4 Cholesterol Treatment Trialists' Collaborators. The effects of lowering LDL cholesterol with statin therapy in people at low risk of vascular disease: meta-analysis of individual data from 27 randomised trials. Lancet 2012; published online 17 May.

5 Jackevicius CA, Chou MM, Ross JS, Shah ND, Krumholz HM. Generic atorvastatin and health care costs. N Engl J Med 2012;366:201-4.

6 Armitage J. The safety of statins in clinical practice. Lancet 2007;370;1781-90.

7 Smeeth L, Douglas I, Hall AJ, Hubbard R, Evans S. Effect of statins on a wide range of health outcomes: a cohort study validated by comparison with randomized trials. Br J Clin Pharmacol 2009;67:99-109.

8 Goldfine $A B$. Statins: is it really time to reassess benefits and risks? N Engl J Med 2012;366:1752-5.

9 Heart Protection Study Collaborative Group, Bulbulia R, Bowman L, Wallendszus K, Parish S, Armitage J, Peto R, et al. Effects on 11-year mortality and morbidity of lowering LDL cholesterol with simvastatin for about 5 years in 20,536 high-risk individuals: a randomised controlled trial. Lancet 2011;378:2013-20.

10 Yusuf S, Zucker D, Peduzzi P, Fisher LD, Takaro T, Kennedy JW, et al. Effect of coronary artery bypass graft surgery on survival: overview of 10-year results from randomised trials by the Coronary Artery Bypass Graft Surgery Trialists Collaboration. Lancet 1994;344:563-70.

Cite this as: BMJ 2012;344:e3802

\section{Related links}

\section{bmj.com}

- bmj.com poll: Should all adults over 50 get statins? Votes: Yes: 189 (21\%); No 704 (79\%)

- Research: Unintended effects of statins in men and women in England and Wales (2010;340:c2197)

- Research: Use of blood pressure lowering drugs in the prevention of cardiovascular disease (2009;338:b1665)

\section{bmj.com/blogs}

- Richard Smith: Statin arguments blog

(c) BMJ Publishing Group Ltd 2012 